\title{
Estratégia\&Negócios
}

ISSN 1984-3372

http://www.portaldeperiodicos.unisul.br/index.php/EeN/

\section{ESTRATÉGIA NO TERCEIRO SETOR: UMA ANÁLISE BIBLIOMÉTRICA E DE CORRELAÇÃO SOBRE A ABORDAGEM ACADÊMICA DO TEMA}

\section{THE THIRD SECTOR STRATEGY: A BIBLIOMETRIC AND CORRELATION ANALYSIS ON THE TOPIC ACADEMIC APPROACH}

\section{Bernardo Paraiso de Campos Serra}

Mestrando pela Faculdade de Economia, Administração e Contabilidade da Universidade de São Paulo - Fea USP. E-mail: bernardoserrari@hotmail.com

\section{Fernanda Cruz Figueiredo}

Mestranda pela Faculdade de Economia, Administração e Contabilidade da Universidade de São Paulo - Fea USP. E-mail: fernanda.figueiredo@usp.br

\section{Martinho Isnard Ribeiro de Almeida}

Professor, Pesquisador e Vice-coordenador do curso de Pós graduação da Faculdade de Economia, Administração e Contabilidade da Universidade de São Paulo - Fea USP.

E-mail: martinho@usp.br

Recebido em 03/12/2012. Aprovado em 01/02/2013. Disponibilizado em 05/05/2013.

Avaliado pelo Sistema double blind review

R. eletr. estrat. neg., Florianópolis, v.6, n.1, p. 229-251, jan./abr.2013

http://portaldeperiodicos.unisul.br/index.php/EeN/index
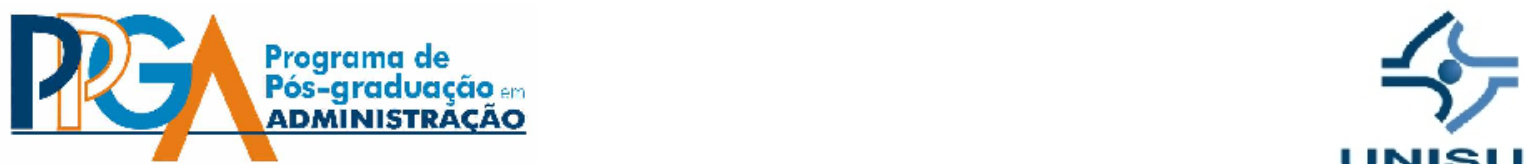

UNISUL

CCopyright 2008 UNISUL-PPGA/Estratégia e Negócios. Todos os direitos reservados. Permitida citação parcial, desde que identificada a fonte. Proibida a reprodução total. Em caso de dúvidas, consulte o editor:

ademar.unisul@gmail.com; (48) 3229-19 


\section{RESUMO}

O propósito do presente artigo é conhecer a produção acadêmica relacionada ao campo da estratégia com foco em organizações do Terceiro Setor. Utilizou-se para este fim o método de pesquisa denominado análise bibliométrica e posteriormente uma análise de correlação. Com as principais palavras-chave relacionadas ao tema, a pesquisa obteve retorno de 105 trabalhos na base de dados Web of Science - ISI Web Of Knowledge. Com isso, foi possível identificar os autores mais referenciados no assunto, as publicações mais relevantes e os principais periódicos, além de construir um gráfico de correlação que mostra as relações entre os trabalhos, identificando tendências dos estudos sobre o tema. Partindo da análise bibliométrica e de correlação, a principal contribuição do trabalho é ilustrar o atual estado da arte sobre o tema e suas lacunas. $O$ trabalho conclui que o tema da estratégia para o Terceiro Setor constitui um amplo campo para futuras pesquisas, e que estudiosos podem aprofundar na questão das peculiaridades dessas organizações para desenvolver uma abordagem mais específica do tema. Sugere-se que sejam feitas também pesquisas complementares em outras bases de dados para corroborar os resultados obtidos.

Palavras-chave: Estratégia. Terceiro Setor. Análise Bibliométrica.

\section{INTRODUÇÃO}

A produção acadêmica em administração passou por diversos estágios. Começando pela fase da administração científica de Taylor, cujo foco era os métodos racionais padronizados de produção, passando pelas teorias clássicas, centradas na estrutura formal da organização e pela escola de relações humanas, que tem enfoque em pessoas, começaram a surgir, em meados da década de 50 , estudos com enfoque na questão do ambiente empresarial.

É no contexto da introdução do conceito de ambiente, que parte da visão da empresa como um sistema aberto que é influenciado e exerce influência sobre seu entorno, que nasce a administração estratégica. A administração estratégica é orientada para o 
ESTRATÉGIA NO TERCEIRO SETOR: UMA ANÁLISE BIBLIOMÉTRICA E DE CORRELAÇÃO SOBRE A ABORDAGEM ACADÊMICA DO TEMA

Bernardo Paraiso de Campos Serra - Fernanda Cruz Figueiredo - Martinho Isnard Ribeiro de Almeida

ambiente, pois, embora atribua significativa relevância aos aspectos internos da organização, considera que o objetivo maior da empresa é fornecer produtos e serviços adequados ao seu ambiente (FERREIRA, 1997).

Considerando que a administração estratégica orienta-se para o futuro e para o atendimento das demandas do ambiente, pode-se dizer que também se faz necessária nas organizações que não visam lucro. Na visão de Hudson (1999), a ideia de criar estratégias explícitas tem se tornado popular no Terceiro Setor porque as organizações têm precisado reagir a mudanças mais rápidas em seu ambiente externo.

Mas, se por um lado as organizações sem fins lucrativos têm certos desafios e metas comuns com as empresas lucrativas, por outro lado, têm peculiaridades e características ambientais próprias que tornam sua administração estratégica particular e um campo específico nos estudos de administração.

Para evoluir nos estudos deste campo, torna-se importante conhecer o estágio atual da produção acadêmica sobre administração estratégica para organizações do Terceiro Setor. Além disso, verifica-se, no terreno prático, um crescimento em número de organizações desse tipo e que cada vez mais elas buscam a profissionalização para garantir sua legitimidade e sustentabilidade, tornando cada vez mais relevante a abordagem do tema.

\section{PROBLEMA DE PESQUISA E OBJETIVOS}

Questão-básica: quais os trabalhos mais referenciados com relação ao tema de estratégia do Terceiro Setor?

Questão-problema: como os trabalhos encontrados nas referências influenciam na formulação dos trabalhos de estratégia com foco no Terceiro Setor?

Assim, o presente estudo tem como objetivos: (a) identificar os autores que são referência no assunto, (b) identificar os trabalhos mais relevantes e (c) identificar os principais periódicos. 


\section{REVISÃO BIBLIOGRÁFICA}

\subsection{ESTRATÉGIA}

Na literatura encontram-se, entre outras, as seguintes definições de estratégia: forma de pensar no futuro, integrada ao processo decisório, com base em um procedimento formalizado e articulador de resultados e em uma programação (MINTZBERG, 1983); busca de uma posição competitiva favorável em uma indústria ou setor (PORTER, 1985); regras e diretrizes para decisão, que orientam o processo de desenvolvimento de uma empresa (ANSOFF, 1990); padrão ou plano que integra os objetivos maiores de uma empresa, suas políticas e sequência de ações em um todo coeso (QUINN, 1992); e planos da alta administração para alcançar resultados consistentes com a missão e os objetivos gerais da organização (WRIGTH, 2000). Assim, a estratégia pode ser definida tanto como plano, padrão, perspectiva ou manobra (MINTZBERG E QUUIN, 2001).

Nos últimos anos, desenvolveram-se paralelamente duas importantes e complementares abordagens sobre estratégia: a visão clássica com foco no mercado externo, por um lado, que parte da posição competitiva da empresa em seu setor para formulação das estratégicas; e a visão baseada em recursos (Resource-based-view), por outro, que tem foco nos recursos internos como principal fonte de vantagem competitiva.

A abordagem clássica da "análise da indústria" ou "posicionamento estratégico" tem Michael Porter (PORTER, 1980) como protagonista principal e considera que a análise dos mercados e a posição relativa de cada empresa em seu setor de atividades são os elementos primordiais para formulação das estratégias. A partir da análise do negócio, dos clientes, das necessidades que se quer atender e dos competidores, a empresa busca a posição estratégica mais favorável. A principal crítica a essa abordagem é de que a preferência dos clientes é volátil e não pode prover um fundamento seguro às estratégias de longo prazo.

A abordagem de RBV, por outro lado, focaliza os recursos internos e considera que cada empresa tem um agrupamento singular de recursos e capacidades que as 
diferencia dos concorrentes e que a formulação das estratégias deve buscar potencializar as competências nas quais a empresa é mais forte.

Na mesma linha da teoria de RBV, Hamel e Prahalad (1990), introduzem o conceito de competências essenciais, que são recursos intangíveis desenvolvidos a partir do aprendizado coletivo da organização, especialmente no modo de coordenar diversas habilidades de produção e de harmonizar fluxos de tecnologias, a organização do trabalho e a entrega de valor. Essa abordagem identifica que as competências essenciais são a raiz da competitividade, as fontes novas e os fundamentos da estratégia.

Tanto a abordagem clássica, considerada de "fora pra dentro", quanto a abordagem de RBV, tida como de "dentro pra fora", foram objeto de pesquisas acadêmicas nos últimos anos e encontraram amplo espaço na literatura sobre estratégia.

\subsection{TERCEIRO SETOR: DEFINIÇÕES}

Terceiro Setor emergiu a partir da mudança do papel do Estado como provedor de bens públicos. Para Tachizawa (2002), após várias décadas de convivência com um Estado que absorvia grande parte das responsabilidades pela provisão de bens e serviços públicos ocorre, atualmente, movimento em direção a minimizar tais encargos e enfatizar a função reguladora do Estado, como decorrência dos novos desafios impostos pelo avanço da privatização.

Ao mesmo tempo, a sociedade civil, que antes participava de ações de desenvolvimento social por meio do exercício do trabalho voluntário em instituições caritativas de igrejas, começa a se organizar e ganhar espaço como um importante agente de promoção social, criando organizações com a finalidade exclusiva de atender uma demanda social. Com caráter privado, essas organizações se propõe atender necessidades de interesse público.

Hudson (1999) define o Terceiro Setor como aquele composto por organizações que: (a) tem basicamente um objetivo social em vez de procurarem gerar lucro; (b) são independentes do Estado porque são administradas por um grupo independente de pessoas 
e não fazem parte de um departamento de governo ou de autoridades locais ou de saúde e (c) reinvestem todo o seu saldo financeiro nos serviços que oferecem ou na própria organização.

Diversas fundações, empresas, organismos multilaterais e governos estão direcionando recursos e buscando parcerias com entidades sem fins lucrativos que promovam projetos de educação, saúde e assistência social e apresentem propostas de efetivas mudanças em comunidades menos favorecidas. Para ter acesso a tais recursos, as organizações do TS têm buscado melhorar seu nível de profissionalização, por meio da utilização de técnicas e modelos de gestão concebidos a partir da realidade do mundo corporativo.

No entanto, existe uma discussão controversa sobre o limite da utilização dessas técnicas e modelos na gestão dessas organizações. Por um lado, considera-se que, assim como as empresas, as organizações sem fins lucrativos necessitam ser eficientes e eficazes para cumprir sua missão, e para isso necessitam utilizar ferramentas de gestão semelhantes. Por outro lado, considera-se que os modelos devem ser adaptados a sua realidade. Para Teixeira (2004), antes de incorporar a lógica e os fundamentos teóricos da Administração tradicional a essas organizações, é necessário discutir contornos da problemática de sua gestão, de maneira que se decida pela elaboração de estudos que considerem a especificidade e as peculiaridades do fenômeno em questão, criando-se fundamentos teóricos mais adequados às características do Terceiro Setor.

\section{METODOLOGIA}

Para o estudo proposto neste artigo, foi feita uma pesquisa bibliométrica, seguido posteriormente de uma análise de correlação. White e McCain (1989) definem bibliometria como o estudo quantitativo das literaturas como são refletidos nas bibliografias. Para a análise de correlação foram criadas matrizes e gráficos que mostram em um espaço de duas dimensões a proximidade de citação entre os trabalhos. Para encontrar a amostra a ser realizada pela pesquisa bibliométrica, foi necessário determinar a base de dados a ser 
ESTRATÉGIA NO TERCEIRO SETOR: UMA ANÁLISE BIBLIOMÉTRICA E DE CORRELAÇÃO SOBRE A ABORDAGEM ACADÊMICA DO TEMA

Bernardo Paraiso de Campos Serra - Fernanda Cruz Figueiredo - Martinho Isnard Ribeiro de Almeida

escolhida, as áreas de estudo dos trabalhos, as palavras-chave a serem utilizadas, e o intervalo de tempo a se pesquisar.

A base de dados escolhida foi a ISI - Web Of Science, por ser uma das mais utilizadas no meio acadêmico. Essa base agrega: Science Citation Index Expanded (SClEXPANDED); Social Sciences Citation Index (SSCl; Arts \& Humanities Citation Index (A\&HCI); e Conference Proceedings Citation Index-Science (CPCI-S). Na pesquisa deste trabalho foram utilizadas todas as bases citadas acima. Com relação ao intervalo de tempo, utilizou-se na pesquisa a opção "All Years".

A lei de Zipf (1949), muito utilizada em estudo bibliométricos, afirma que a frequência de aparecimento de uma determinada palavra é inversamente proporcional a sua posição na tabela de frequência, ou seja, a palavra na primeira posição é duas vezes mais citada que a segunda, três vezes mais que a terceira, e assim por diante. Levando em conta a Lei de Zipf e com o intuito de maximizar os resultados encontrados, foi feito um estudo bibliográfico pré-pesquisa para encontrar as palavras-chave mais utilizadas em trabalhos de estratégia com foco em Terceiro Setor, conforme quadro 1.

Quadro 1 - Palavras-chave utilizadas na pesquisa

\begin{tabular}{|l|l|}
\hline Palavras-chave utilizadas: \\
\hline nonprofit strategy & supporting-organization strategic planning \\
\hline nonprofit strategic planning & charity-organization strategy \\
\hline non-profit strategy & charity-organization strategic planning \\
\hline non-profit strategic planning & community-organization strategy \\
\hline third-sector strategy & community-organization strategic planning \\
\hline third-sector strategic planning & NGO strategy \\
\hline noncommercial strategy & NGO strategic planning \\
\hline noncommercial strategic planning & social-enterprise strategy \\
\hline non-commercial strategy & social-enterprise strategic planning \\
\hline non-commercial strategic planning & philanthropic-organization strategy \\
\hline supporting-organization strategy & philanthropic-organization strategic planning \\
\hline
\end{tabular}

Fonte: Elaborado pelos autores, (2012).

Terminada a pesquisa inicial com as palavras-chave, os resultados foram filtrados, selecionando na página de resultados apenas áreas de estudo que eram de interesse para a pesquisa: Management e Business. Posteriormente, os resultados foram 
exportados da base Web Of Science e salvos para futura análise. É importante ressaltar que a pesquisa foi efetuada no início de maio de 2011 e, seguindo os passos acima, foram encontrados 105 trabalhos.

Levou-se em conta também a Lei de Bradford, que afirma que a quantidade de periódicos de um determinado campo de estudo podem ser divididas em 3 partes. Uma quantidade pequena de " $x$ " periódicos são responsáveis por $1 / 3$ das publicações; uma segunda leva maior de periódicos determinada por " $x$ " multiplicado por uma constante " $n$ " é responsável por mais 1/3; e um restante de periódicos determinados por " $x$ " multiplicado

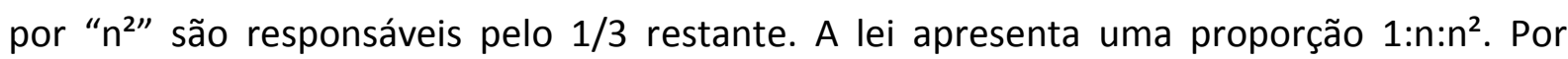
exemplo, em um campo de estudo com 365 periódicos e um total de 1500 publicações, 5 periódicos detêm 500 (1/3) das publicações de um campo de estudo, 40 periódicos (x.n = 5 vezes 8) detêm outras $500(1 / 3)$ e finalmente 320 periódicos $\left(x \cdot n^{2}=5\right.$ vezes 8 elevado ao quadrado) detém mais 500 (1/3).

Partindo dos pressupostos acima e com o auxílio de um software bibliométrico chamado BibExcel, foi organizado o seguinte conteúdo: tipos de publicação, trabalhos mais citados nas referências dos artigos, periódicos dos artigos encontrados com maior número de publicações, autores mais citados nas referências dos artigos, criação das matrizes de correlação de referências.

Com as matrizes de correlação prontas foi feita a criação dos gráficos de correlação de referências por meio do software SPSS. Em um segundo momento, foi feita uma análise dos trabalhos mais citados nas referências e dos gráficos de correlação das referências mais citadas a fim de entender como os trabalhos citados influenciaram na formulação dos artigos encontrados, que se referem à questão da estratégia aplicada ao Terceiro Setor.

\section{ANÁLISE DOS RESULTADOS}

Como parte dos resultados, tem-se a Tabela 1 abaixo, na qual se encontra discriminado o total e os tipos de trabalhos encontrados na pesquisa. 
ESTRATÉGIA NO TERCEIRO SETOR: UMA ANÁLISE BIBLIOMÉTRICA E DE CORRELAÇÃO SOBRE A ABORDAGEM ACADÊMICA

Tabela 1 - Tipos de Trabalhos

\begin{tabular}{l|l}
\hline Quantidade: & Tipo de trabalho: \\
\hline 74 & Artigo \\
\hline 21 & Trabalho de congresso \\
\hline 9 & Revisão \\
\hline 1 & Nota \\
\hline 105 & Total \\
\hline
\end{tabular}

Fonte: Elaborado pelos autores, (2012).

Pela pesquisa foi possível identificar também quais os periódicos com maior quantidade de trabalhos publicados, conforme Tabela 2.

Tabela 2 - Periódicos com mais trabalhos publicados

\begin{tabular}{l|l|l}
\hline Qtd.: & Periódico: & Frequência \\
\hline 4 & Journal of Management Studies & $3,81 \%$ \\
\hline 4 & Journal of Business Ethics & $3,81 \%$ \\
\hline 3 & European Journal of Marketing & $3,81 \%$ \\
\hline 3 & Journal of Business Research & $2,86 \%$ \\
\hline 3 & British Journal of Management & $2,86 \%$ \\
\hline 3 & Strategic Management Journal & $2,86 \%$ \\
\hline 3 & Long Range Planning & $2,86 \%$ \\
\hline 2 & Journal of Organizational Change Management & $2,86 \%$ \\
\hline 2 & Technological Forecasting And Social Change & $1,90 \%$ \\
\hline 2 & Management Decision & $1,90 \%$ \\
\hline 2 & Journal of Business Venturing & $1,90 \%$ \\
\hline 2 & Journal of Management \& Organization & $1,90 \%$ \\
\hline 2 & Public Management Review & $1,90 \%$ \\
\hline 2 & Journal of Management & $1,90 \%$ \\
\hline 2 & Journal of Operations Management & $1,90 \%$ \\
\hline 2 & Business \& Society & $1,90 \%$ \\
\hline 2 & California Management Review & $1,90 \%$ \\
\hline Fon & Elaborado pelos autores, (2012). & $1,90 \%$ \\
\hline 3 &
\end{tabular}

Fonte: Elaborado pelos autores, (2012).

Pela análise da frequência dos periódicos, verificou-se que aproximadamente 45\% dos trabalhos encontrados foram publicados em 18 periódicos, conforme Tabela 3, o 


\section{ESTRATÉGIA NO TERCEIRO SETOR: UMA ANÁLISE BIBLIOMÉTRICA E DE CORRELAÇÃO SOBRE A ABORDAGEM ACADÊMICA DO TEMA}

Bernardo Paraiso de Campos Serra - Fernanda Cruz Figueiredo - Martinho Isnard Ribeiro de Almeida

que permite concluir que se tratam dos mais relevantes para pesquisa sobre o tema do Terceiro Setor.

\subsection{TRABALHOS MAIS CITADOS NAS REFERÊNCIAS E O QUE ABORDAM}

A Tabela 3 mostra as referências mais citadas dos trabalhos encontrados.

Tabela 3 - Referências mais citadas nos artigos encontrados

\begin{tabular}{|c|c|c|}
\hline $\begin{array}{l}\text { Qtde. de } \\
\text { Citações }\end{array}$ & Trabalho & Frequência \\
\hline 12 & $\begin{array}{l}\text { EISENHARDT, K. M. Building theories from case study research. The academy of } \\
\text { management review, v. } 14, \text { n. } 4 \text {, p. } 532-550,1989 .\end{array}$ & $11,43 \%$ \\
\hline 9 & $\begin{array}{l}\text { FREEMAN, R. E Strategic Management: A Stakeholder Approach New York, McGraw Hill, } \\
\text { 1984. }\end{array}$ & $8,57 \%$ \\
\hline 7 & $\begin{array}{l}\text { DIMAGGIO, P. J.; POWELL, W. W. The Iron Cage Revisited: Institutional Isomorphism and } \\
\text { Collective Rationality in Organizational Fields. American Sociological Review, v. 48, n. 2, p. } \\
\text { 147, } 1983 .\end{array}$ & $6,67 \%$ \\
\hline 7 & $\begin{array}{l}\text { BARNEY, J. Firm resources and sustained competitive advantage. Journal of management, } \\
\text { v. } 17, \text { n. } 1, \text { p. } 99,1991 \text {. Sage Publications. }\end{array}$ & $6,67 \%$ \\
\hline 6 & $\begin{array}{l}\text { MITCHELL, R. K.; AGLE, B. R.; WOOD, D. J. Toward a theory of stakeholder identification and } \\
\text { salience: Defining the principle of who and what really counts. The Academy of } \\
\text { Management Review, v. } 22, \text { n. } 4 \text {, p. } 853-886,1997 .\end{array}$ & $5,71 \%$ \\
\hline 6 & $\begin{array}{l}\text { DOH, J. P.; TEEGEN, H. Nongovernmental organizations as institutional actors in } \\
\text { international business: theory and implications. International Business Review, v. 11, n. } 6 \text {, } \\
\text { p. } 665-684,2002 \text {. }\end{array}$ & $5,71 \%$ \\
\hline 6 & HANSMANN, H. The role of nonprofit enterprise. Yale Law Journal, v. 89, p. 835-902, 1980. & $5,71 \%$ \\
\hline 6 & $\begin{array}{l}\text { PORTER, M. E. Competitive advantage: creating and sustaining competitive performance. } \\
\text { New York: Free Press, } 1985 .\end{array}$ & $5,71 \%$ \\
\hline 5 & $\begin{array}{l}\text { WERNERFELT, B. A resource-based view of the firm Strategic Management Journal V. 5, no } \\
2,171-180 \text {, abril-junho } 1984 \text {. }\end{array}$ & $4,76 \%$ \\
\hline 5 & $\begin{array}{l}\text { BERGER, I.; CUNNIGNGHAM, P.; DRUMWRIGHT, M. Social alliances: company/nonprofit } \\
\text { collaboration. California Management Review, v. 47, n. 1, p. 58-90, } 2004 .\end{array}$ & $4,76 \%$ \\
\hline 5 & $\begin{array}{l}\text { GLASER, B. G; STRAUSS, A. L. The Discovery of Grounded Theory: Strategies for Qualitative } \\
\text { Research. New York: Aldine Publishing Company, } 1967 .\end{array}$ & $4,76 \%$ \\
\hline 5 & $\begin{array}{l}\text { TEEGEN, H.; DOH, J. P.; VACHANI, S. The importance of nongovernmental organizations } \\
\text { (NGOs) in global governance and value creation: an international business research } \\
\text { agenda. Journal of International Business Studies, v. } 35, \text { n. } 6, \text { p. } 463-483,2004 .\end{array}$ & $4,76 \%$ \\
\hline 5 & $\begin{array}{l}\text { SPAR, D. L.; LA MURE, L. T. The power of activism: Assessing the impact of NGOs on global } \\
\text { business. California Management Review, v. 45, n. 3, p. 78-101, } 2003 .\end{array}$ & $4,76 \%$ \\
\hline 5 & $\begin{array}{l}\text { STINCHCOMBE, A. L Social Structure and Organizations. In: J. G. March (Ed.), Handbook, of } \\
\text { Organizations (pp. 142-193). Chicago, IL: Rand McNally \& Company.1965. }\end{array}$ & $4,76 \%$ \\
\hline 5 & PFEFFER, J.; SALANCIK, G. R. The external control of organizations. New York, v. 263, 1978. & $4,76 \%$ \\
\hline
\end{tabular}

Fonte: Elaborado pelos autores, 2012. 
O trabalho Eisenhardt (1989) trata do método de Estudo de Caso, utilizado para abordar casos específicos a serem estudados, dissecando em especial o caso em que se estuda, sem generalizar as conclusões obtidas.

O trabalho de Freeman (1984) aborda a teoria de Stakeholders, que é voltada para a gestão organizacional e ética de negócios. Diferentemente da visão comum de que empresas que tem como objetivo atender os interesses de seus investidores, empregados, fornecedores, e clientes, a teoria de Stakeholders considera que existem diversas outras partes envolvidas, como por exemplo, grupos políticos, comunidades, e o público em geral. A teoria defende também que a empresa deve levar em consideração seus valores internos na criação de suas estratégias, e que isso, juntamente com os valores de seus gestores e as expectativas dos stakeholders em geral, irá determinar o sucesso da empresa.

Barney (1991) trata da teoria de RBV (Resource Based View), que aborda a vantagem competitiva de uma empresa como reflexo de seus recursos. $\mathrm{O}$ trabalho apresenta um método para a empresa avaliar se seus recursos são realmente fonte de vantagem competitiva, que consiste basicamente em dizer se são valiosos, raros de se encontrar, difíceis de imitar, e explorados corretamente pela organização.

O artigo de Dimaggio e Powell (1983), fundadores da escola do neoinstitucionalismo, trata do isomorfismo, que significa que organizações inseridas em um mesmo ambiente tornam-se cada vez mais semelhantes ao longo do tempo, afetando a capacidade de mudança ao longo do tempo. Os autores entendem que as causas da burocratização citadas por Weber mudaram e passaram a ser outras, ou seja, as empresas competem não somente por recursos e consumidores, mas por poder político e legitimidade institucional. Assim, os gestores das organizações tomam decisões não apenas estratégicas do ponto de vista de mercado e recursos, mas também com intuito político, acarretando no longo prazo em mimetismo organizacional.

Mitchell et al. (1997) em seu trabalho também abordam a teoria de Stakeholders, complementando com uma tipologia de stakeholders baseada nos atributos poder, legitimidade, e urgência. Esses atributos combinados geram perfis de stakeholders, cada um com uma implicação específica para a organização. 
O trabalho de Doh e Teegen (2002) parte da teoria de Stakeholders de Freeman (1984) e aborda a influência das organizações de Terceiro Setor na formulação e adoção de políticas no campo laboral e ambiental que regulem as atividades de empresas multinacionais. Os autores desenvolvem também uma teoria para explicar o porquê de as organizações de Terceiro Setor terem maior ou menor influência no processo de desenvolvimento e aceite de condutas normativas internacionais, citando Mitchell et al (1997), ao adotar os conceitos de poder, legitimidade e urgência como explicação para sua teoria.

Hansmann (1980) discute o conceito, o papel e as características essenciais das organizações sem fins lucrativos sob uma perspectiva econômica. $\mathrm{O}$ autor sugere que as organizações sem fins lucrativos são uma espécie de resposta razoável a um "fracasso do mercado", uma falha no contrato entre o consumidor e o mercado, ou como uma espécie de controle sobre as empresas de um determinado setor. O Terceiro Setor teria sua origem no estabelecimento de um novo vínculo tentando suprir falhas de mercado, ou como um supervisor regulando o mercado, em prol da sociedade.

Porter (1980), em seu livro Competitive Strategy, apresenta um posicionamento estratégico baseado em Structure-Conduct-Performance ou SCP. O autor afirma que a estrutura em que se compete, juntamente com sua conduta no ambiente competitivo irão moldar a performance. Para analisar a estrutura do mercado e escolher a conduta, o autor apresenta o Modelo das Cincos Forças e das Estratégias Genéricas, dando à organização a possibilidade de utilizar de frameworks para posicionar-se em seu ambiente competitivo.

O trabalho de Wernerfelt (1984), um dos primeiros artigos de RBV, apresenta recursos como fontes de forças ou fraquezas da empresa, bem como vantagens competitivas. Discute ainda de onde surgem os recursos de uma empresa, e quais recursos podem ser úteis em diferentes mercados.

O artigo de Berger et al. (2004) aborda a aliança entre empresas sem fins lucrativos e empresas com fins lucrativos, englobando os problemas previsíveis nas alianças e as dimensões em que devem se encaixar com a do parceiro, a estrutura das empresas, e a estrutura das alianças. Quanto aos problemas previsíveis nas alianças, cita que podem ser 
mal-entendidos, alocação ruim de custos e recursos, desbalanceamento de poder, incompatibilidades dos parceiros, problemas com tempo e falta de confiança. Para suprir tais problemas, entende que deve haver o encaixe da missão, dos recursos, da gestão, dos colaboradores internos, do mercado alvo, do produto/causa, da cultura, do tempo e da avaliação dos benefícios da aliança.

O trabalho de Grounded Theory de Glaser (1967) refere-se a uma metodologia em que se pesquisa e se colhem os dados para posteriormente construir uma teoria, visando a melhor adaptação da teoria ao conteúdo do trabalho.

Teegen et al. (2004) discutem o surgimento das organizações de Terceiro Setor como participantes importantes na criação de valor e na governança global. Abordam a definição e a dinâmica do campo institucional, a relevância/centralização do modelo de barganha da organização e a organização dentro do ambiente de atuação.

Spar e La Mure (2003) tratam do modo como as ONGs exercem pressão sobre as empresas e como elas respondem a esse ativismo. No artigo os autores demonstram também como as empresas respondem ao ativismo e o que levam em conta ao tomar suas decisões: os custos da tomada de decisão, a visão da sociedade em relação à decisão, e se o impacto da decisão na vantagem competitiva da empresa é benéfico ou não.

Stinchcombe (1965) dá ênfase em como a estrutura do ambiente influencia as empresas no momento de sua criação. O ambiente vigente no momento da criação da organização tende a influenciar tanto as estruturas básicas das empresas, como suas estratégias, sendo que esses efeitos tendem a se perpetuar pela inércia das empresas ao longo do tempo.

Ainda na linha da influência do ambiente, Pfeffer e Salancink (1978) defendem que as organizações sobrevivem à medida que elas são efetivas, e essa efetividade origina-se do gerenciamento das demandas dos grupos de interesse com os quais a organização estabelece uma relação de dependência de recursos e apoio, e de sua habilidade de criar resultados e ações aceitáveis por eles. 
ESTRATÉGIA NO TERCEIRO SETOR: UMA ANÁLISE BIBLIOMÉTRICA E DE CORRELAÇÃO SOBRE A ABORDAGEM ACADÊMICA DO TEMA

Bernardo Paraiso de Campos Serra - Fernanda Cruz Figueiredo - Martinho Isnard Ribeiro de Almeida

\subsection{A ANÁLISE DO GRÁFICO DE CORRELAÇÃO}

Partindo da Tabela 4 foi elaborado um gráfico de correlação entre os trabalhos, conforme apresentada na Figura 1. O gráfico tem por objetivo mostrar visualmente a proximidade de citação entre os trabalhos: quanto mais próximos os pontos, mais vezes os trabalhos foram citados juntos, e quanto maior o círculo, mais vezes o trabalho foi citado o nas referências.

Figura 1 - Gráfico de referências

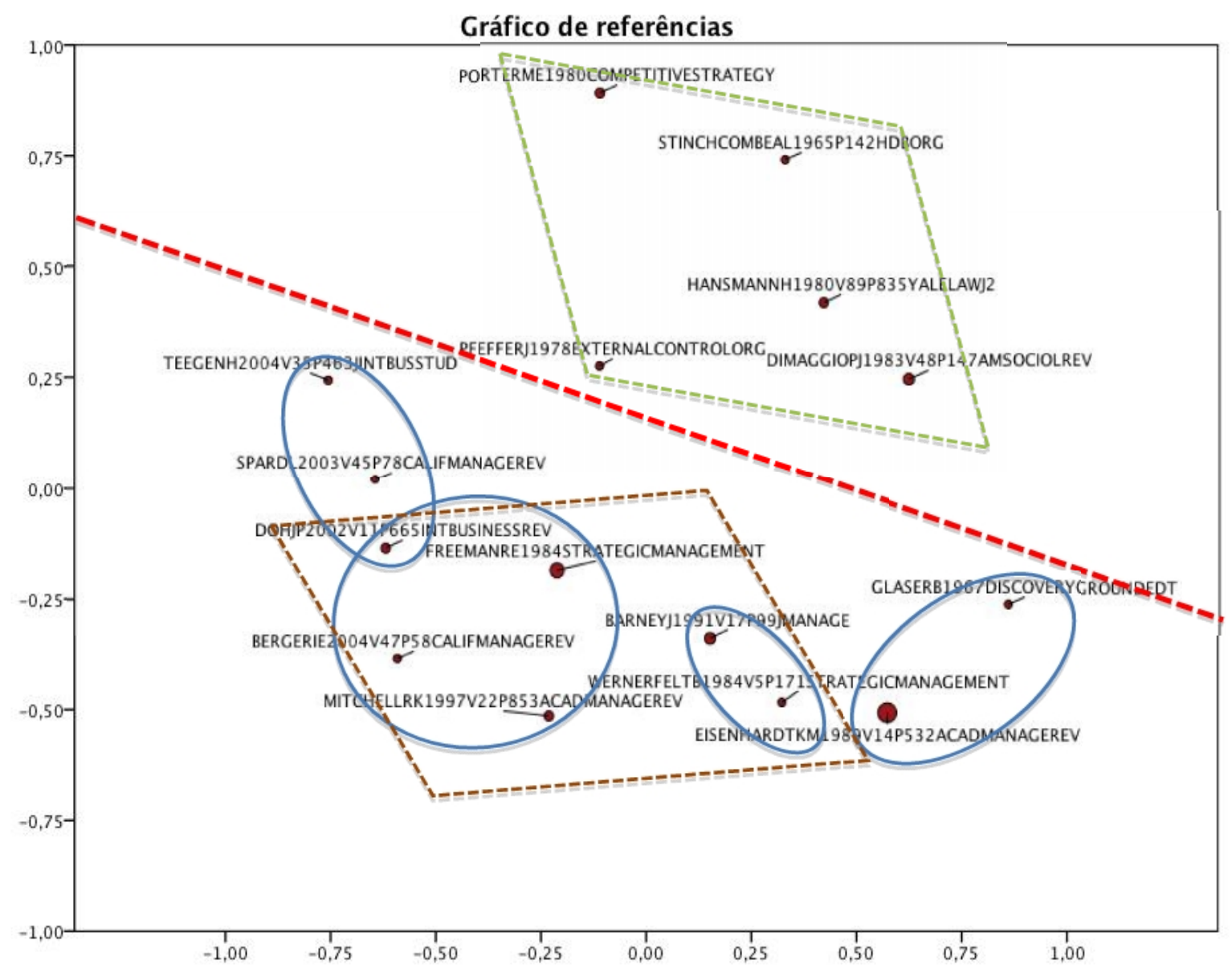

Fonte: Elaborado pelos autores, 2012.

Examinado o gráfico, primeiramente é possível ver um agrupamento por métodos. A citação do trabalho de Eisenhardt (1989), no caso o mais citado nas referências, 
sugere que existe uma grande tendência a se abordar o estudo do Terceiro Setor por meio de estudo de caso. Já a citação do trabalho de Grounded Theory de Glaser (1967) aponta uma tendência a se pesquisar e colher os dados para posteriormente escolher qual teoria irá se adaptar melhor ao conteúdo do trabalho.

Em segundo ponto é possível identificar um cluster com Barney e Wernerfelt como representantes da teoria de RBV.

No terceiro agrupamento se encontram autores da teoria de Stakeholders como Freeman, Mitchell, Berger e Doh. Freeman e Mitchell tratam explicitamente a teoria de Stakeholders, que aborda a necessidade de se ajustar seus valores e recursos internos, ao mesmo tempo em que se tem uma preocupação com seus stakeholders. Já Doh e Berger se apropriam da teoria de Stakeholders para justificarem seus trabalhos.

Existe uma interação entre o agrupamento de RBV com o agrupamento da teoria de stakeholders, no ponto em que os trabalhos presentes no agrupamento de stakeholders fundamentam-se em recursos da organização, juntamente com um foco em stakeholders, para então analisar como as organizações influenciam alianças, regulamentos e políticas externas.

Um quarto agrupamento é o que contém Teegen, Spar e Doh, que abordam a questão de como organizações de Terceiro Setor exercem pressão em governos, e empresas com fins lucrativos.

A linha pontilhada vermelha marca uma cisão na abordagem de interpretação, a metade inferior foca nos recursos internos, e como eles podem influenciar a empresa e a sociedade em geral. Já a metade superior define a organização como sendo extremamente suscetível ao ambiente externo, com o foco do desempenho da empresa determinado pelo setor em que está inserida, e sua atuação nesse setor.

O último agrupamento é dos que focam o resultado da empresa como sendo determinado pelo ambiente externo, incluindo Porter e seu modelo baseado em EstruturaConduta-Performance; Stinchcombe e a influência do ambiente na formulação da empresa; Hansmann e sua teoria das ONGs como criadas para serem lacunas de mercado e controladoras em prol da sociedade; DiMaggio e o ambiente como força de pressão política, 
ESTRATÉGIA NO TERCEIRO SETOR: UMA ANÁLISE BIBLIOMÉTRICA E DE CORRELAÇÃO SOBRE A ABORDAGEM ACADÊMICA DO TEMA

Bernardo Paraiso de Campos Serra - Fernanda Cruz Figueiredo - Martinho Isnard Ribeiro de Almeida

gerando a isomorfia; e Pfeffer com a abordagem que a organizações são influenciadas por fatores do ambiente, que geram as relações de dependência.

\subsection{AUTORES MAIS CITADOS NAS REFERÊNCIAS E SUAS CONTRIBUIÇÕES AO TEMA}

Ainda como contribuição do presente trabalho, a Tabela 4 mostra os autores mais citados dos trabalhos encontrados.

Tabela 4 - Autores mais citados nos artigos encontrados

\begin{tabular}{l|l|l}
\hline & $\begin{array}{l}\text { Autores mais citados nas } \\
\text { Quantidade de citações: }\end{array}$ & Frequência de aparecimento nos artigos: \\
\hline 39 & Drucker, P. F. & $37,14 \%$ \\
30 & Mintzberg, H. & $28,57 \%$ \\
24 & Kotler, P. & $22,86 \%$ \\
22 & Nutt, P. C. & $20,95 \%$ \\
21 & Porter, M. E. & $20,00 \%$ \\
20 & Eisenhardt, K. M. & $19,05 \%$ \\
20 & Kaplan, R. S. & $19,05 \%$ \\
17 & Doh, J. P. & $16,19 \%$ \\
15 & Yin, R. K. & $14,29 \%$ \\
14 & Austin, J. E. & $13,33 \%$ \\
13 & Gulati, R. & $12,38 \%$ \\
13 & Bryson, J. M. & $12,38 \%$ \\
13 & Pfeffer, J. & $12,38 \%$ \\
12 & Anheier, H. K. & $11,43 \%$ \\
\hline
\end{tabular}

Fonte: Elaborado pelos autores, 2012.

Drucker dedicou boa parte de sua produção acadêmica ao estudo de organizações do Terceiro Setor. Em seu livro Administração de Organizações Sem Fins Lucrativos: Princípios e Práticas (1997), por exemplo, aponta que essas organizações têm peculiaridades que merecem atenção: sua missão, que tanto as distingue das empresas e dos governos e que traduz o seu "resultado", as estratégias necessárias para comercialização de seus serviços e a obtenção do dinheiro necessário para execução da sua tarefa e o desafio de introduzir mudanças e inovações pelo fato de trabalharem essencialmente com voluntários. 
Kotler aparece, graças a sua contribuição acerca do Marketing Social, abordado em diversos de seus livros. Em um de seus últimos livros publicados, Marketing 3.0, reforça a importância dos aspectos sociais no Marketing e aborda que este passou por três fases, estando agora em sua $3^{\text {a }}$ fase, logo, o Marketing 3.0 (KOTLER, 2010). Nas palavras do próprio Kotler (2010, p.53), no Marketing 3.0 "seu objetivo é fornecer soluções para problemas da sociedade... o Marketing 3.0 complementa o Marketing emocional com o Marketing do espírito humano."

Nutt, pesquisador da área de estratégia, dedica alguns trabalhos a tratar dos contornos da administração estratégica em organizações públicas e de Terceiro Setor. O autor tenta explicar as principais características dos três setores com relação ao ambiente, transações e processos organizacionais e entende que as diferenças entre eles fazem com que as abordagens tradicionais sobre estratégia, desenvolvidas para o setor privado, sejam inadequadas para organizações públicas (NUTT, 1993).

Kaplan, um dos criadores do Balanced Scorecard, escreve um trabalho específico, em que aplica essa ferramenta na avaliação da performance de organizações sem fins lucrativos, explicando que devem ser avaliadas pela capacidade de atender as demandas da sociedade, de arrecadar recursos e controlar despesas (KAPLAN, 1996).

Doh aborda a questão da ascensão do papel das organizações do Terceiro Setor no cenário internacional. Buscando fundamento na Teoria Institucional e na Teoria de Stakeholders, o autor mostra a importância das ONGs no cenário político-econômico global (DOH; TEEGEN, 2002; TEEGEN et al., 2004) e de que forma as diferentes estruturas institucionais dos países explicam como governos e ONGs influenciam as ações de Responsabilidade Social das Empresas (DOH; GUAY, 2006).

Austin é especialista em alianças e responsabilidade social e escreve também sobre assuntos como as diferenças entre empreendedorismo social e comercial (AUSTIN et al., 2006), a importância das parcerias entre os diferentes setores (BARRETT et al., 2000) e as lideranças no Terceiro Setor (AUSTIN, 1998).

Gulati, especializado em redes e alianças, aborda em seus trabalhos questões como formação, escolha da estrutura de governança, evolução, performance das alianças 
(GULATI, 1998) e do aprendizado (KHANNA; GULATI, 1998; GULATI, 1999). Embora os trabalhos do autor abordem o tema de maneira ampla, são muito utilizados como fundamento teórico para a abordagem sobre alianças e parcerias intersetoriais entre empresas, governos e organizações do Terceiro Setor.

Uma contribuição teórica importante ao tema do planejamento estratégico específico para Terceiro Setor é apresentada por Bryson (1988), que apresenta um modelo de Planejamento Estratégico para organizações públicas e para organizações sem fins lucrativos. O autor afirma que os modelos de planejamento estratégico para essas organizações devem considerar como formular e implementar estratégias em diferentes situações: ser explicitamente político, indicar como lidar com diferentes, ambíguos e conflitantes metas e objetivos; indicar como se deve lidar com a colaboração e a competitividade e estabelecer papéis para o planejamento estratégico.

Pffefer, além de desenvolver a teoria da dependência dos recursos (PFEFFER; SALANCIK, 1978) tem pesquisas também no campo do poder de gestão (PFEFFER; GROUP, 1992) e desenvolveu a abordagem da gestão baseada em evidências, que propõe que as empresas devem encontrar e aplicar as melhores práticas de gestão para suas empresas, que copiar cegamente modelos que parecem ter funcionado em outros lugares (PFEFFER; SUTTON, 2006).

Finalmente, Porter e Mintzberg não são citados por trabalhos relacionados especificamente ao Terceiro Setor, mas aparecem por serem referências no assunto estratégia. Estando o modelo das 5 forças (PORTER, 1980), e o conceito de estratégia emergente (MINTZBERG, 2001), entre os conceitos mais importantes desses autores para o estudo de organizações do Terceiro Setor.

\section{CONCLUSÕES}

A partir da pesquisa bibliométrica utilizando as palavras-chave relacionadas aos temas de Estratégia e Terceiro setor, foram apresentadas as principais bases utilizadas em estudos acadêmicos sobre o assunto. Verificou-se, pelos trabalhos mais referenciados, 
ESTRATÉGIA NO TERCEIRO SETOR: UMA ANÁLISE BIBLIOMÉTRICA E DE CORRELAÇÃO SOBRE A ABORDAGEM ACADÊMICA DO TEMA

Bernardo Paraiso de Campos Serra - Fernanda Cruz Figueiredo - Martinho Isnard Ribeiro de Almeida

algumas tendências como o uso dos métodos de pesquisa Estudos de Caso e Groudend Theory, a busca de fundamentação em teorias de stakeholders, de ambiente e de alianças e também a ampla utilização dos trabalhos e autores clássicos de Estratégia.

Verificou-se também que existe um esforço no sentido desenvolver teorias de gestão e estratégia que contemplem a realidade das organizações do TS, inclusive por parte de autores que tradicionalmente abordam o tema sob a perspectiva de empresas. No entanto, muitas dessas tentativas procuram adaptar modelos criados para o mundo corporativo à realidade dessas organizações, sem aprofundar na questão das suas peculiaridades que justificam a abordagem específica do tema. Assim, futuras pesquisas podem explorar a questão das peculiaridades dessas organizações para construção de modelos e teorias mais adequadas a sua realidade.

A principal limitação da pesquisa foi a utilização de uma única base de dados. Recomenda-se, para estudos posteriores, o uso de mais fontes para confrontar a base utilizada e garantir maior confiabilidade dos dados. Também pode ser considerada uma limitação o baixo número de retorno de artigos na pesquisa bibliométrica, pois isso torna mais difícil fazer inferências sobre o estado da arte do tema.

\section{THE THIRD SECTOR STRATEGY: A BIBLIOMETRIC AND CORRELATION ANALYSIS ON THE TOPIC ACADEMIC APPROACH}

\section{ABSTRACT}

The purpose of this article is to know related to the academic field of strategy-focused organizations in the Third Sector. It was used for this purpose the research method called bibliometric analysis and subsequently a correlation analysis. With the main keywords related to the topic, the survey achieved a return of 105 works in the database Web of Science - ISI Web Of Knowledge. Thus, it was possible to identify the most referenced authors in the field, the most relevant publications and the mainstream media as well as developing a correlation graphic showing the relationship between the work, identifying the 
ESTRATÉGIA NO TERCEIRO SETOR: UMA ANÁLISE BIBLIOMÉTRICA E DE CORRELAÇÃO SOBRE A ABORDAGEM ACADÊMICA DO TEMA

Bernardo Paraiso de Campos Serra - Fernanda Cruz Figueiredo - Martinho Isnard Ribeiro de Almeida

trends of studies on the subject. Starting from the bibliometric analysis and correlation, the main contribution of this work is to illustrate the current state of the art on the theme and its gaps. The paper concludes that the theme of the strategy for the Third Sector is a broad field for future research, and that scholars can deepen the question of the peculiarities of these organizations to develop a more specific theme. It is suggested that further research be done also in other databases to corroborate the results obtained.

Key-Words: Strategy. Third Sector. Bibliometric Analysis.

\section{REFERÊNCIAS}

ANSOFF, I. Estratégia empresarial. São Paulo: Atlas, 1990.

AUSTIN, J. Business Leaders and Nonprofits. Nonprofit Management and Leadership, v. 9, n. 1, p. 39-52, 1998.

AUSTIN, J.; STEVENSON, H.; WEI-SKILLERN, J. Social and commercial entrepreneurship: same, different, or both? Entrepreneurship Theory and Practice, v. 30, n. 1, p. 1-22, 2006.

BARNEY, J. Firm resources and sustained competitive advantage. Journal of management, $v$. 17, n. 1, p. 99, 1991. Sage Publications.

BARRETT, D.; AUSTIN, J.; MCCARTHY, S. Cross-sector collaboration: lessons from the International Trachoma Initiative. Havard Business School - social enterprise series, n. 16, 2000.

BERGER, I.; CUNNIGNGHAM, P.; DRUMWRIGHT, M. Social alliances: company/nonprofit collaboration. California Management Review, v. 47, n. 1, p. 58-90, 2004.

BRYSON, J. A. A Strategic Planning Process for Public and Non-profit Organizations. Long Range Planning, v. 21, n. 1, 1988.

DIMAGGIO, P. J.; POWELL, W. W. The Iron Cage Revisited: Institutional Isomorphism and Collective Rationality in Organizational Fields. American Sociological Review, v. 48, n. 2, p. 147, 1983.

DOH, J. P.; TEEGEN, H. Nongovernmental organizations as institutional actors in international business: theory and implications. International Business Review, v. 11, n. 6, p. 665-684, 
ESTRATÉGIA NO TERCEIRO SETOR: UMA ANÁLISE BIBLIOMÉTRICA E DE CORRELAÇÃO SOBRE A ABORDAGEM ACADÊMICA DO TEMA

Bernardo Paraiso de Campos Serra - Fernanda Cruz Figueiredo - Martinho Isnard Ribeiro de Almeida

2002.

DOH, J. P.; GUAY, T. R. Corporate Social Responsibility, Public Policy, and NGO Activism in Europe and the United States: An Institutional-Stakeholder Perspective. Journal of Management Studies, v. 43, n. 1, p. 47-73, 2006.

DRUCKER, P. F. Administração de Organizações Sem fins Lucrativos. Princípios e Práticas. São Paulo: Pioneira, 1997.

EISENHARDT, K. M. Building theories from case study research. The academy of management review, v. 14, n. 4, p. 532-550, 1989.

FERREIRA, A. A. Gestão empresarial: de Taylor aos nossos dias: evolução e tendências da moderna administração de empresas São Paulo: Pioneira. 1997.

FISCHMANN, Adalberto A e ALMEIDA, Martinho I. R. Planejamento estratégico na prática. São Paulo, Atlas, 1991.

FREEMAN, R. E Strategic Management: A Stakeholder Approach New York, McGraw Hill, 1984.

GLASER, B. G; STRAUSS, A. L. The Discovery of Grounded Theory: Strategies for Qualitative Research. New York: Aldine Publishing Company, 1967.

GULATI, R. Alliances and networks. Strategic Management Journal, v. 19, p. 293-317, 1998.

GULATI, R. Network location and learning: The influence of network resources and firm capabilities on alliance formation. Strategic management journal, 1999.

HAMEL, G.; PRAHALAD, C. K. The core competence of the corporation. Harvard business review, v. 68, n. 3, p. 79-91, 1990.

HANSMANN, H. The role of nonprofit enterprise. Yale Law Journal, v. 89, p. 835-902, 1980.

HUDSON, M. Administrando Organizações do Terceiro Setor: O Desafio de Administrar Sem Receita. São Paulo: Makron Books, 1999.

KAPLAN, R. S.; NORTON, D. P. Using the balanced scorecard as a strategic management system. Harvard business review, v. 74, n. 1, p. 75-85, 1996.

KHANNA, T.; GULATI, R. The dynamics of learning alliances: competition, cooperation, and relative scope. Strategic Management Journal, v. 19, n. 3, p. 193-210, 1998. 
ESTRATÉGIA NO TERCEIRO SETOR: UMA ANÁLISE BIBLIOMÉTRICA E DE CORRELAÇÃO SOBRE A ABORDAGEM ACADÊMICA DO TEMA

Bernardo Paraiso de Campos Serra - Fernanda Cruz Figueiredo - Martinho Isnard Ribeiro de Almeida

KOTLER, P; KARTAJAYA, H.; SETIAWAN, I. Marketing 3.0: As forças que estão definindo o novo marketing centrado no ser humano. Rio de Janeiro: Elsevier, 2010.

MINTZBERG, H. What is planning anyway. Strategic Management Journal, New York, no 2, oct. 1983.

MINTZBERG, H.; AHLSTRAND, B.; LAMPEL, J. Strategy safari: A guided tour through the wilds of strategic management. Free $\mathrm{Pr}, 2005$.

MINTZBERG, H.; QUINN, J. B. O processo da estratégia. 3. ed. Porto Alegre: Bookman, 2001.

MITCHELL, R. K.; AGLE, B. R.; WOOD, D. J. Toward a theory of stakeholder identification and salience: Defining the principle of who and what really counts. The Academy of

Management Review, v. 22, n. 4, p. 853-886, 1997.

NUTT, P. Organizational publicness and its implications for strategic management. Journal of Public Administration Research and, v. 3, n. 2, 1993.

PFEFFER, J.; SALANCIK, G. R. The external control of organizations. New York, v. 263, 1978.

PFEFFER, J.; GROUP, S. V. M. Managing with power. Harvard Business School Press, 1992.

PFEFFER, J.; SUTTON, R. I. Evidence-based management. Harvard business review, v. 84, n. 1, p. 62-74, 133, 2006.

PORTER, M. E. Competitive strategy: techniques for analysing industrie and competitors. New York : Free Press, 1980.

PORTER, M. E. Competitive advantage: creating and sustaining competitive performance. New York: Free Press, 1985.

QUEIROZ, M. O Planejamento Estratégico e as Organizações do Terceiro Setor. In: Voltolini, R. (Org.) Terceiro Setor: Planejamento e Gestão. São Paulo: SENAC São Paulo, 2004.

QUINN, James B. Intelligent entreprise. New York: The Free Press, 1992.

SPAR, D. L.; LA MURE, L. T. The power of activism: Assessing the impact of NGOs on global business. California Management Review, v. 45, n. 3, p. 78-101, 2003.

STINCHCOMBE, A. L Social Structure and Organizations. In: J. G. March (Ed.), Handbook, of Organizations (pp. 142-193). Chicago, IL: Rand McNally \& Company.1965.

TACHIZAWA, T. Organizações Não-Governamentais e Terceiro Setor: criação de ONGs e Estratégias de Atuação. São Paulo: Atlas, 2002.

R. eletr. estrat. neg., Florianópolis, v.6, n.1, p. 229-251, jan./abr.2013

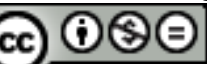


ESTRATÉGIA NO TERCEIRO SETOR: UMA ANÁLISE BIBLIOMÉTRICA E DE CORRELAÇÃO SOBRE A ABORDAGEM ACADÊMICA DO TEMA

Bernardo Paraiso de Campos Serra - Fernanda Cruz Figueiredo - Martinho Isnard Ribeiro de Almeida

TEEGEN, H.; DOH, J. P.; VACHANI, S. The importance of nongovernmental organizations (NGOs) in global governance and value creation: an international business research agenda. Journal of International Business Studies, v. 35, n. 6, p. 463-483, 2004.

TEIXEIRA, R.F. Discutindo o Terceiro Setor sob o enfoque de concepções tradicionais e inovadoras de administração. Cadernos de pesquisas em Administração, São Paulo, v. 11, n. 1, p. 1-15, janeiro-março de 2004.

1 WERNERFELT, B. A resource-based view of the firm Strategic Management Journal v. 5, no 2, 171-180, abril-junho 1984.

WHITE, H. D.; MCCAIN, K. W. Bibliometrics. Annual review of information science and technology, v. 24, p. 119-186. Information Today, 1989.

WRIGHT, P., KROLL, M., PARNELL, J.. Administração Estratégica. São Paulo: Atlas, 2000.

ZIPF, George K. Human Behavior and the Principle of Least Effort. Cambridge, Massachusetts: Addison-Wesley, 1949. 